DOI: https://doi.org/10.24297/jap.v16i1.8483

\title{
The Inverse Precise Placement of Numbers Based at Pythagoras 1:3 And The Precise Configuration of Numbers as The Basis of A Unified Theorem that Binds the Physics of Mass, Space, Energy and The Torque Power(Inertia) that Sustains the Finite Universe. (Section 11, Physics)
}

\author{
Vinoo Cameron \\ Hope Clinic, Athens, Wisconsin \\ hope9900@frontier.com
}

\begin{abstract}
This paper is based on the precise inverse cone of Pythagoras 1:3. As in section 1 of this paper, all mathematics presented in this paper is by precise mathematics equations and the author has maintained by proof that the base numbers constant from which all physics constants can be derived are -1 to 19 ( the value 6 is as per this paper is the constant for expansion of all bounded space and 19 is the patent "end value" of the base constant numbers as shown in section 1 and referenced in this section11). Numbers as created and as placed at the cone of Pythagoras 1:3 are precise manifestation of the numbers of linear composite. However, this paper shows that the invention of designated angles (Trigonometry) is an approximate arbitrary arrangement invented by man, based on the created fixed angle of 90 degrees and is certainly flawed as shown here in this paper. Likewise, any attempts to measure curvature by linear numbers is fraught with much error. The author maintains that "If the atomic density and structure of meteorites from far space have the same configuration as those found on this earth, then by all created logic, these numbers configuration presented in these two papers and the book (The God of Papa Einstein and Sir Isaac Newton) are a constant unified theorem of all the space and mass ( Quantum mechanics) in the universe". Einstein's Theory of relativity and all other interstellar phenomena are not addressed in the context of this paper because all this is observed science phenomena and not a physical science, and this paper deals with precise numbers configuration as in the section 1 of the paper. Einstein's relativity is a real observed natural phenomenon, not a science by itself, it is a natural aberration of the fact that observed relativity is due to inherent curvature and linear relationship between any two points in the universe and because of the spiral progression of curved space. Neither does light bend it appears to bend, nor does time really dilate in real terms even though it is a real observed phenomena, neither numbers or distances dilate by any continuum, unfortunately that is why Einstein's misconception about time dilation is a Theory and will always be Theory.
\end{abstract}

Keywords: Inverse Cone of Pythagoras 1:3, Constant Value 6, Katie's Equation, Inertia

1.The precise configuration of expansion of all bound space constant at the value 6 as a base constant for the minimum and maximal bound space as defined by 1:3 Pythagoras (Katies equation as discovered by the Author).

Reference diagram 1, (The 1X6 at Pythagoras 1:3)

By Katies equation the configuration of Pythagoras 1:3.

$(X+Y=1 /(X-Y)$

$(X-Y)=1 /(X+Y)$

1:3 Pythagoras cone (base is 3 , hypotenuse is $\sqrt{ } 10$ )

$(3+\sqrt{ } 10)=1 /(3-\sqrt{ } 10)=-6.16227766017$ 
$(19+\sqrt{ } 360)=1 /(19-\sqrt{ } 360)=37.97366596103$

$6.16227766017^{\wedge} 2=37.97366596103$.

(Note: In the above equalization $3^{\wedge} 2+\mathbf{1}=10$ and $19^{\wedge} 2-\mathbf{1}=360$. This determines the root of $1: 3$ as -1 )

Absolute precision of equation

$1 / 6.16227766017=0.16227766017$ Katie's (6.16227766017-6)

$1 / 37.97366596103=0.02633192204$

$(0.97366596103+0.02633192204=1)$

$37.97366596103-0.02633192204=\sqrt{ } 360 * 2$

$\sqrt{ } 10 * 6=\sqrt{ } 360$

Thus, by Pythagoras theorem: the maximal constant value of $1: 3$ is $6: 18$

The span of the equation is 6

Whereas $3^{\wedge} 2+1^{\wedge} 2=\sqrt{ } 10$

$18^{\wedge} 2+6^{\wedge} 2=\sqrt{ } 360$

$(3+\sqrt{ } 10) \wedge 2-19=\sqrt{ } 360$

\section{The representation of the 5,6 curve constants at the exclusive whole number curve constant of 1:6}

\section{Reference diagram 2 (5,6-curve constant)}

The 1:6 curve constant at radius 5 is the only exclusive whole number curve in the entire geometry along with the basic $1: 2$ curve of a circle $(6 / 2=3)$, as discovered by the author. The precise configuration of the 5,6 radius/ span is parallel to all prime numbers and their derivatives as the standard division by the constant 6 , residua $(1 / 6),(5 / 6)$. Please note this precise configuration based on Pythagoras theorems in the following precise mathematical resolutions.

a. The reference diagram as shown in this context is based on the Pythagoras theorem.

b. The radius is 5 and the span is precise 6 , with a precise height of the curve at value 1 , the only whole number curve at 1 and 6 curves in the entire geometry of the universe, aside from the 1:2 curve of a basic circle at radius 1 .

c. Note the sagittal placed 1:3 cones that comprise the 1:6 curve.

d. Note the Pythagorean value $3^{\wedge} 2+4^{\wedge} 2=\sqrt{ } 25(5)$

e. Note the precise representation of 5,6 as $1 / 6$ and $5 / 6$ the precise residua when prime numbers and their derivatives the pseudo prime numbers when divided by constant value 6 as derived from Katie's equation at Pythagoras 1:3 as shown above.

f. Note that the angle subtended at $1: 3$ in the diagram (360/19) is precisely one fourth $(1 / 4)$ of the angle subtended at the center by the span of 6 . There are 4 such whole angles plus 0.75 of 4(3) 3 at the 6/5.3223. In precise composite $4 * 4+3=19$

g. Note that the designated value of the angle at 1:3 (360/19 degrees) is at variance from the current Trigonometric value of 18.44 degrees. The author maintains that current trigonometry is in error because it presumes that all divisions of angles are rationally divisible which they are not. There is further mathematical proof of 360/19 in the text of the book. Moreover, the clear proportionate designated values of the angles 
subtended at the center $(4 * 360 / 19)$ as shown precisely in the diagram is $4 * 4+3=19$. The value $360 / 19(X)$ is the only designated value in degrees that satisfies the equation $16 X+3 x=360$ degrees.

h. Note that the precise value 5.322 at the diagram. This incidentally and directly led to the constant $6 / 5.322(1.1273957159)$ that is shown in section 1 of the papers as the "precise digital phenomena at prime number $7 "$ which is the twin prime with prime number 5 .

i. Note that the 1:6 curve is the value of the fixed curved and linear relationship between any two points in the universe space, clearly defining the matrix of the universe space being inherently "curvilinear at any two given points" in the universe space.

3.The Inherent Torque force (inertia) in the universe, manifested at the particle level (the inherent electromagnetism at particle level)

This is a clear precise resolution of and by physics based on the absolute linear at the half line of the cone (1:3) and the spiral tangent placement of numbers resulting in a predictable outer and inner spiral orientation as shown in section 1. This is clear, this is the so called "Torque force" at particle level even in dark energy of all mass as the indestructible so called "electro-magnetic force" at the atomic level. This same understanding was reached independently in a caveat of the paper that the author read at JAP when his Section1 paper was published in the same edition of the paper by Author Leonard Van Zanten (cited under reference. ) The author notes that he has found fresh and pragmatic articles in the chaos of these pragmatic journals like JAP, rather than the elite journals that are straight jacketed by their own dogmatic understanding of scientific progress ". The author has duly informed some of these elite journals of their stymied approach to new research as one notes that even after one hundred years of the discovery of Max Plank's constant there has not been any real credible progress in the advent of scientific discovery and understanding in the understanding of the universe and we have confused theories like the String theory and Einstein's observational theory of Relativity.

Conclusion and discussion: The author have laid down the basics of physics and mathematics by the unified spiral placement of numbers at 1:3 Pythagoras and the value 19. The universe is inherently curved and linear at any two given points (1:6 curve constant). All phenomena of science of the universe including mass, gravity, energy) are easily defined by this precise configuration of numbers, by resolution based on Pythagoras 1:3 and base number constants. Einstein's relativity is an observed science not a reality of physics ( Time only appears to dilate and light only appears to bend but created numbers never dilate from their base -1 to 19 ). The Max Plank number constant by digits is a derivative of 6 constant $(6.62607015$, and this value is fully divisible by $3,5,6,7$ as a closed value and by their derivatives $21,35,105,210)$. All the basis of all physics constants as it is shown in section 1 and section 11 of this paper, as a rule of mathematical physics, the base constant numbers (-1to19) must follow this created dictum based on Pythagoras 1:3 and note the fact that the universe is curved/ linear at any two given points. Mass and energy are defined by numbers and their inherent torque force between the linear and the curve as is shown in the spirals and the constant exclusive 1:6 curve (curve-linear at any two given points in the universe). The author has constrained his work to the standard of Pythagoras 1:3 and proved that numbers are created at a fixed spiral arrangement. All future research must be based on this fact of creation that is mentioned in the scriptures such as the Holy Bible and the "Guru Nanak's Grunth" of the Sikhs, and other scriptures which the author has not studied. The author happens to be of an Anglo- Indian- Sikh heritage which is an element of chance as no race or ethnicity is special in the created sense, although "Truth is singular" as we know it; it is evident in some of the scriptures of mankind and cannot be removed from the universe. God is one indeed, as the sole creator of all science.

\section{References:}

1. Journal of Advances in Physics vol 16 (2019) ISSN: 2347-3487: The Inverse Infinite Spiral Placement of Prime Numbers and their Derivatives at The Cone of Pythagoras 1:3. By Vinoo Cameron.

2. Journal of Advances in Physics vol 16 (2019) ISSN: 2347-3487.Law of Gravity, by Leonard Van Zanten End. 